\section{P115 SOCIO-DEMOGRAPHIC PROFILE OF HIV SEROPOSITIVITY IN A TERTIARY CARE NORTH INDIAN HOSPITAL: A TEN YEAR RETROSPECTIVE STUDY}

${ }^{1}$ Sarbjeet Sharma, ${ }^{1}$ Gagandeep Singh, ${ }^{2}$ Nikhil Sharma*. 'Sgrdimsar, Microbiology, Amritsar, India; ${ }^{2}$ Guru Nanak Dev University, Mass Communication and Journalism, Amritsar, India

10.1136/sextrans-2019-sti.298

Background All over the world, with 36.9 million people living with HIV/AIDS, the latter continues to be a major public health problem having tremendous social and economic impact. However, increasing global awareness and availability of effective treatment and prevention programmes, promise a hope of controlling the pandemic.

Methods Serum samples of 53,357 clients who attended ICTC, Sri Guru Ram Das University of Health Sciences (SGRDUHS), Amritsar, Punjab (India) from January 2007 to December 2017, were tested for HIV infection as per NACO guidelines using 3 kits with different antigen preparation/test principles viz. Comb AIDS RS, Meriscreen HIV -1-2 WB \& Signal-HIV after a pre-test counseling \& taking informed consent.

Results Of the total 53,357 clients tested for HIV infection, $630(1.2 \%)$ were found to be HIV positive.Seropositivity was higher in males 387/630(61.42\%) compared to females 243/ 630(38.57\%) although females 29911/53357(56.05\%) outnumbered the males 23426/53357(43.9\%) in voluntary HIV testing. No transgender visited our ICTC during this period. Heterosexual route was the major route of transmission seen in 507/630 clients (80.47\%). Main age group affected was $35-49$ years $224 / 630(35.55 \%)$, followed by $25-34$ years $185 /$ $630(29.36 \%),>50$ years $107 / 630(16.98 \%), 15-24$ years $77 /$ $630(12.22 \%) \&<15$ years only 37/630(5.9\%).

Conclusion Barring 2011, total number of clients tested each year has shown an ascending trend which can be attributed to increasing global awareness about the disease, decreasing associated stigma, expanded media coverage and the widespread availability of anti-retroviral therapy (ART). Although overall prevalence is low at $1.2 \%$, IDU prevalence saw two peaks viz. $33.33 \%(4 / 12)$ in 2007 and 20\%(14/70) in 2012 and currently $8.2 \%(4 / 49)$ in 2017 puts the spotlight on HIV burden in this risk group and suggests the need for the scaling up of focused preventive efforts in high-risk groups.

Disclosure No significant relationships.

\section{P122 TREATMENT OUTCOMES IN HIV CARE AMONG CHILDREN AND ADULT IN HIGH VOLUME ART SITES IN RIVERS STATE, NIGERIA}

Atochi Emenike* Rivers State Ministry of Health, Public Health, Port Harcourt, Nigeria

\subsection{6/sextrans-2019-sti.299}

Background The HIV program involves sequential steps: HIV testing, diagnosis, linkage to care, retention and viral-suppression. Access to Antiretroviral-Therapy with appropriate regimen, continuity of care, technical competence of service providers on counselling and client focus are required to meet the bench mark for quality and standards of care. Most often than not, retention in care of People Living with HIV is a public health issue. This study aims to review retention and variations in treatment outcomes among adult and children in high-volume ART sites in Rivers State Nigeria

Methods This is a retrospective cohort-study investigating oneyear treatment outcomes of adults (15 years and above) and children (0-14 years) who started ART between January 2017-December 2017 across 6 high-volume ART sites in Rivers State Nigeria, supported by the USAID funded SIDHAS project and Global Fund. A treatment current of 1000 and above was the rationale for choosing the high-volume ART sites. The study involved secondary data collection, using the Retention-Audit-Determination-Tool (RADET).

Results A total of 3704 records of individuals who started antiretroviral therapy were reviewed, $4.7 \%$ (177) and $96.5 \%$ (3577) accounted for children and adult respectively. Treatment outcomes after one-year on treatment showed that $61.0 \%$ (108) of children were active, $2.8 \%$ (5) dead, $19.2 \%$ (34) transferred-out and $35.6 \%$ (63) lost-to-follow-up. Adults had $80.8 \%$ (2891) active outcome, $1.3 \%$ (48) dead, $2.7 \%$ (99) transferred-out, $12.2 \%$ (437) Lost-to-Follow-Up and $0.3 \%$ (12) stopped-treatment

Conclusion Positive treatment-outcome is important for viralload suppression, reduced morbidity and mortality. Transferred-Out is considerably high among children and even more for Lost-to-Follow-Up outcome among children. We found variation in attrition between children and adults. Higher mortality rate was recorded among children as compared to adults. A well-planned children and adult counselingmethods, follow-up tracking, family-centered approach and client understanding of lifelong-ART are required in Patient Management, Monitoring to improve treatment-outcomes for PLHIV especially children on ART

Disclosure No significant relationships.

\section{P123 HIV SEROPOSITIVITY AND EARLY SYPHILIS STAGE PREDICTIVE OF OCULAR SYPHILIS DIAGNOSIS IN BRITISH COLUMBIA, CANADA: 2010-2018}

${ }^{1}$ Hasan Hamze* ${ }^{2}$ Venessa Ryan, ${ }^{2}$ Emma Cumming, ${ }^{1}$ Christine Lukac, ${ }^{2}$ Jason Wong, ${ }^{2}$ Troy Grennan. 'University of British Columbia, Faculty of Medicine, Vancouver, Canada; ${ }^{2} B C$ Centre For Disease Control, Vancouver, Canada

\subsection{6/sextrans-2019-sti.300}

Background The incidence of syphilis has been increasing worldwide in the last 20 years, and disproportionately affecting people living with HIV. Additionally, several jurisdictions have reported increasing incidence of ocular syphilis (OS). If untreated or treatment is delayed, OS can lead to permanent blindness. We assessed characteristics of OS cases in British Columbia (BC), and sought to identify risk factors that may be associated with OS diagnosis, including factors related to HIV co-infection (e.g. CD4 count. viral load).

Methods This case-control study matched OS cases to syphilis controls (1:4) diagnosed in BC between January 2010 March 2018. Demographic and clinical data were extracted from the provincial sexually transmitted infection database and analyzed using the chi-squared test. A multivariate logistic model was developed to assess factors associated with OS. 